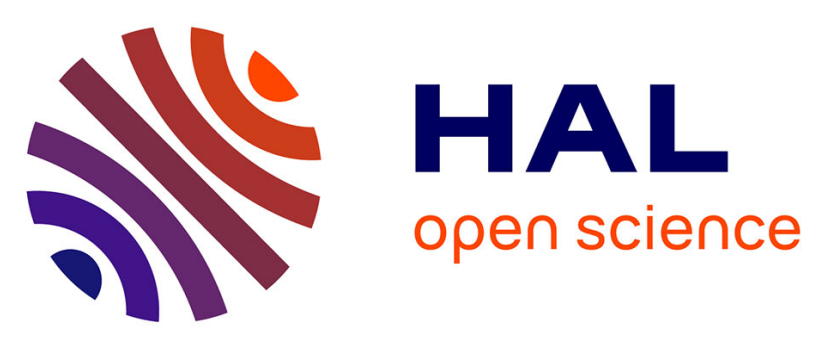

\title{
Chronic NOS inhibition prevents adverse lung remodelling and pulmonary arterial hypertension in caveolin-1 knockout mice
}

Carsten Wunderlich, Alexander Schmeisser, Christian Heerwagen, Bernd Ebner, Kristin Schober, Ruediger C. Braun-Dullaeus, Carsten Schwencke, Michael Kasper, Henning Morawietz, Ruth H. Strasser

\section{To cite this version:}

Carsten Wunderlich, Alexander Schmeisser, Christian Heerwagen, Bernd Ebner, Kristin Schober, et al.. Chronic NOS inhibition prevents adverse lung remodelling and pulmonary arterial hypertension in caveolin-1 knockout mice. Pulmonary Pharmacology \& Therapeutics, 2008, 21 (3), pp.507. 10.1016/j.pupt.2007.11.005 . hal-00499152

\section{HAL Id: hal-00499152 https://hal.science/hal-00499152}

Submitted on 9 Jul 2010

HAL is a multi-disciplinary open access archive for the deposit and dissemination of scientific research documents, whether they are published or not. The documents may come from teaching and research institutions in France or abroad, or from public or private research centers.
L'archive ouverte pluridisciplinaire HAL, est destinée au dépôt et à la diffusion de documents scientifiques de niveau recherche, publiés ou non, émanant des établissements d'enseignement et de recherche français ou étrangers, des laboratoires publics ou privés. 


\section{Author's Accepted Manuscript}

Chronic NOS inhibition prevents adverse lung remodelling and pulmonary arterial hypertension in caveolin-1 knockout mice

Carsten Wunderlich, Alexander Schmeisser, Christian Heerwagen, Bernd Ebner, Kristin Schober, Ruediger C. Braun-Dullaeus, Carsten Schwencke, Michael Kasper, Henning Morawietz, Ruth H. Strasser

PII: $\quad$ S1094-5539(07)00102-2

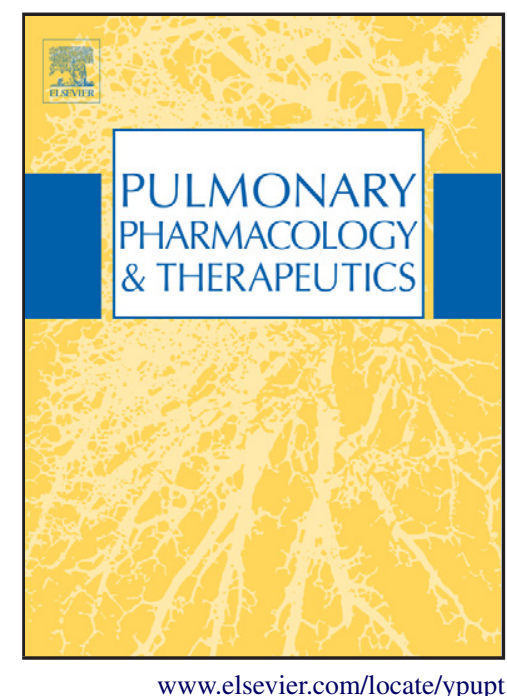

DOI: $\quad$ doi:10.1016/j.pupt.2007.11.005

Reference: $\quad$ YPUPT 807

To appear in: Pulmonary Pharmacology \& Therapeutics

Received date: 26 July 2007

Revised date: 8 November 2007

Accepted date: 21 November 2007

Cite this article as: Carsten Wunderlich, Alexander Schmeisser, Christian Heerwagen, Bernd Ebner, Kristin Schober, Ruediger C. Braun-Dullaeus, Carsten Schwencke, Michael Kasper, Henning Morawietz and Ruth H. Strasser, Chronic NOS inhibition prevents adverse lung remodelling and pulmonary arterial hypertension in caveolin-1 knockout mice, Pulmonary Pharmacology \& Therapeutics (2007), doi:10.1016/j.pupt.2007.11.005

This is a PDF file of an unedited manuscript that has been accepted for publication. As a service to our customers we are providing this early version of the manuscript. The manuscript will undergo copyediting, typesetting, and review of the resulting galley proof before it is published in its final citable form. Please note that during the production process errors may be discovered which could affect the content, and all legal disclaimers that apply to the journal pertain. 


\title{
Chronic NOS inhibition prevents adverse lung remodelling and pulmonary arterial hypertension in caveolin-1 knockout mice
}

\author{
Carsten Wunderlich $^{1 *}$, Alexander Schmeisser ${ }^{1^{*}}$, Christian Heerwagen $^{1}$, Bernd Ebner ${ }^{1}$, \\ Kristin Schober ${ }^{1}$, Ruediger C. Braun-Dullaeus ${ }^{1}$, Carsten Schwencke ${ }^{1}$, Michael Kasper ${ }^{2}$, \\ Henning Morawietz ${ }^{3}$, and Ruth H. Strasser ${ }^{1}$
}

${ }^{1}$ University of Technology Dresden, Department of Cardiology, Medical Clinic, Fetscherstr. 76, Dresden 01307, Germany.

${ }^{2}$ Department of Anatomy, Fiedlersr. 42, Dresden 01307, Germany

${ }^{3}$ Department of Microcirculation, Fiedlerstr. 42, Dresden 01307, Germany

* both authors equally contributed to this work

Address for correspondence:

Carsten Wunderlich M.D.

Department of Medicine and Cardiology

University of Technology Dresden

Fetscherstr. 76

01307 Dresden, Germany

Tel.: $\quad+493514501700$

Fax.: $\quad+493514501702$

Email: carstenwunderlich@gmx.de

Manuscript information: 22 text pages, 5 figures and 1 table.

Word count: Abstract, 239; total manuscript, 4733

Abbreviations: eNOS-endothelial nitric oxide synthase, wt-wild type, cav-1 ko-caveolin-1 knockout animals, L-NAME - $\mathrm{N}^{\mathrm{G}}$-nitro-L-arginine methyl ester 
Wunderlich et al. NOS-inhibition prevents lung remodelling in caveolin-1 knockout mice

\section{Abstract}

Recently generated caveolin-1 deficient mice (cav-1 ko) suffer from severe lung fibrosis with marked pulmonary hypertension and arterial hypoxemia and may therefore serve as an useful animal model of this devastating human disorder. Accumulating evidence strongly supports the negative regulatory influence of caveolin-1 on endothelial nitric oxide synthase resulting in a constitutive hyperactivation of the nitric oxide (NO) pathway in cav-1 ko. We therefore hypothesized that a disturbed NO signalling is implicated in the evolution of the adverse lung phenotype of cav-1 ko. For this purpose cav-1 ko of 2 month age were compared with knockout counterparts experiencing 2 month postnatal NO synthase inhibition by $\mathrm{N}^{\mathrm{G}}$-nitro-Larginine methyl ester (L-NAME) treatment. Chronic L-NAME administration prevented adverse lung remodelling in cav-1 ko. Furthermore, L-NAME donation led to a normalized oxygen saturation $(91.5 \pm 1.8$ vs. $98.5 \pm 2.3 \%, \mathrm{P}<0.01, \mathrm{n}=10-12)$, a marked decrease in right ventricular hypertrophy (LV/RV ratio: $4.0 \pm 0.3$ vs. $2.7 \pm 0.3, \mathrm{P}<0.01, \mathrm{n}=10-12)$ and reductions of the elevated pulmonary artery pressure $(40.2 \pm 3.1$ vs. $26.3 \pm 4.6 \mathrm{mmHg}, \mathrm{P}<0.01, \mathrm{n}=6)$. Collectively, these improvements resulted in an enhanced exercise capacity of L-NAME treated cav-1 ko. Finally, we found evidence for enhanced oxidative stress in untreated cav-1 ko which was substantially reduced by chronic L-NAME administration to cav-1 ko. In view of these data we speculate that a perturbation of $\mathrm{NO}$ signalling, together with enhanced $\mathrm{O}_{2}{ }^{-}$ production originating from NO synthases, may play a pivotal role in the pathogenesis of the adverse pulmonary phenotype seen in cav-1 ko.

Key words: caveolin, eNOS, nitric oxide, superoxide, lung fibrosis 
Wunderlich et al. NOS-inhibition prevents lung remodelling in caveolin-1 knockout mice

\section{Introduction}

Caveolae are small invaginations of the plasma membrane abundantly present in most cells of the cardiopulmonary system. In these cells caveolae function in protein trafficking, cholesterol homeostasis and signal transduction. Three family members of caveolins are identified to date. Caveolin-1 (cav-1) and caveolin-2 (cav-2) are coexpressed in adipocytes, fibroblasts and endothelial cells while caveolin-3 (cav-3) expression is confined to muscle cells. ${ }^{1}$ Cav-1 and cav-3 are essential for the biogenesis of caveolae in cells that normally express these isoforms ${ }^{2-4}$, whereas cav-2 is not absolutely required. ${ }^{5}$

Recently, the caveolin proteins have been implicated in structural lung remodelling and development of pulmonary arterial hypertension $(\mathrm{PAH}) .{ }^{5-8}$ Indeed, one prominent feature of caveolin-1 knockout mice (cav-1 ko) is an adverse lung phenotype with thickened alveolar septa, smaller alveolar spaces and an interstitial hypercellularity. ${ }^{2 ; 3}$ Cav-1 ko are further characterized by a substantial pulmonary arterial hypertension (PAH) which is accompanied by a hypertrophied right ventricle (RV). ${ }^{9}$ Moreover, cav-1 ko were shown to suffer from a dramatic reduction in life span. ${ }^{10}$ Of note, cav-1 ko also display a near complete ablation of cav-2 expression. These findings provide the basis for an intense debate on the functional implications of cav-1 on lung remodelling. ${ }^{8}$ However, the most direct evidence for a independent role of cav-1 on pulmonary function versus alveolar thickening is supported by data documenting that recently generated cav-2 deficient mice (with retained cav-1 expression) do not have PAH, but do exhibit pulmonary hyperplasia. ${ }^{5}$ Moreover, several papers reported a decreased expression of pulmonary caveolins in various animal models of $\mathrm{PAH}^{8 ; 11}$ These findings also appear to be relevant to human PAH in that decreases in cav-1 gene and protein expression have been recently demonstrated in plexiform lesions of patients

with severe $\mathrm{PAH} .{ }^{12}$ Altogether these studies validate the importance of caveolins in the development of lung pathologies but the specific role of cav-1 in this process awaits further clarification. 
Wunderlich et al. NOS-inhibition prevents lung remodelling in caveolin-1 knockout mice

Within the vascular endothelium endothelial nitric oxide synthase (eNOS) targets to caveolae of the plasma membrane where it is tonically inhibited by interaction with cav-1 resulting in a constitutive hyperactivation of the nitric oxide (NO) pathway in cav-1 ko.;9;13 There is mounting evidence that eNOS can be converted to an important 'reactive oxygen species' generator within the vessel wall. ${ }^{14,15}$ Within the so-called "uncoupled" eNOS enzyme electrons flow to molecular oxygen and generate superoxide $\left(\mathrm{O}_{2}^{-}\right)$instead of NO. This scenario results in a reduced NO production by eNOS while this enzyme additionally produces $\mathrm{O}_{2}^{-}$. The superoxide anion avidly reacts with vasodilating $\mathrm{NO}$ to form the oxidant species peroxynitrite. ${ }^{16}$ Accordingly, the NO bioavailability is further reduced with concurrent increased oxidant stress.

$\mathrm{PAH}$ is a common finding in human lung fibrosis. ${ }^{17}$ Despite recent advances in symptomatic treatment this disease is characterized by a progressive malfunction associated with a poor prognosis of affected individuals. Unfortunately, no current treatment option cures this devastating condition. Various molecules have been implicated as putative candidates of its pathogenesis including endothelial dysfunction due to impaired NO signalling. ${ }^{17}$ In this context recently generated cav-1 ko may serve as an useful model of this disorder and help to further unveil the molecular mechanism of this disease and to uncover potential targets for future therapies.

Given the adverse pulmonary phenotype of cav-1 ko and the close regulatory interaction between cav-1 and eNOS in normal physiology we questioned whether a perturbation of NO signalling with consecutive enhanced oxidative stress may be involved in the genesis of the pathological lung phenotype in cav-1 ko. Therefore we treated respective animals with the NO synthase inhibitor $\mathrm{N}^{\mathrm{G}}$-nitro-L-arginine methyl ester (L-NAME) for 2 month and studied the exercise capacity, pulmonary function, lung morphology and the $\mathrm{O}_{2}{ }^{-}$production in related tissues thereafter. 
Wunderlich et al. NOS-inhibition prevents lung remodelling in caveolin-1 knockout mice

\section{Materials and Methods}

\section{Animals}

Age and gender matched mice were bred and housed in a barrier facility at the Institute for Animal Studies of the University of Technology, Dresden, Germany. After birth one group of animals (cav-1 ko and age/gender matched wild-type littermates) received standard care with a normal rodent chow and drinking water ad libitum. A second group was additionally treated with oral L-NAME (50 mg/kg, dissolved in drinking water) beginning after birth. Mice of two months age were included in the study. Animal experiments conformed to the Guide for the Care and Use of Laboratory Animals published by the US National Institutes of Health (NIH Publication No. 85-23, revised 1996). The generation of the transgenic mice has been described elsewhere. $^{3}$

\section{Hemodynamic measurements}

Mice were injected with 250 units heparin intraperitoneally (i.p.) and anesthetized by injection of $12 \mathrm{mg} / \mathrm{kg}$ xylazine i.p. and $80 \mathrm{mg} / \mathrm{kg}$ ketamine i.p. and placed on a warming table to keep the body temperature at $37^{\circ} \mathrm{C}$ throughout the experiment. Subsequent to anesthesia, the mice were orally intubated, connected to a rodent ventilator (TSE Animal Respirator, Bad Homburg, Germany) and ventilated in a pressure-controlled manner at a rate of 110 breaths per minute with the fraction of inspired oxygen being $21 \%$. After shaving and disinfection a home-made saline filled catheter connected to a pressure transducer (ADInstruments, Powerlab, MLT844, Spechbach, Germany) was inserted into the jugular vein and advanced to the pulmonary artery for hemodynamic studies. The central venous pressure and the pulmonary artery pressure was evaluated at end expiration. Simultaneously, the left ventricular end diastolic pressure (LVEDP) was measured with a home-made saline filled catheter connected to a pressure transducer (ADInstruments, Powerlab, MLT844, Spechbach, 
Wunderlich et al. NOS-inhibition prevents lung remodelling in caveolin-1 knockout mice

Germany) after retrograde passage of the aortic valve. We applied the following formula for calculation of the transpulmonary gradient (TPG=PAPmean-LVEDP). All data were measured continuously by using a PC with dedicated software (ADInstruments, Chart, Spechbach, Germany).

\section{Blood analysis}

After anesthesia and injection with Heparin (250 units i.p.), the mice were ventilated in a pressure-controlled manner at a rate of 110 breaths/min only with room air. A midline skin incision at the lower xiphoid was performed, and the skin was retracted laterally. Afterwards, a substernal abdominal midline incision was done to approach the abdominal surface of the diaphragm, which then was incised to visualize the hearts apex. The oxygenated blood was collected from left ventricle with a heparin-covered syringe. The hemoglobin and hematocrit content and the oxygen saturation were measured with a Micros 60 blood analyzer (Horiba Diagnostics, Irvine, California, USA).

\section{Lung and liver weight}

For determination of the lung weight, fresh lungs were flushed in saline to remove contaminating blood and weighed immediately (wet weight). The lungs were then placed in a drying oven at $80^{\circ} \mathrm{C}$ until a constant weight was obtained (dry weight). Tissue water content was determined according to the following formula [(wet wt- dry wt/ wet wt) $x$ 100]. For measurement of the liver weight the organ was harvested, flushed in saline to remove contaminating blood and weighed immediately (liver weight). To normalize differences in lungs/liver weight which can be attributed to the overall size of the mouse we then choose to express the results in relation to the total body weight. 
Wunderlich et al. NOS-inhibition prevents lung remodelling in caveolin-1 knockout mice

\section{Assessment of right ventricular hypertrophy}

The heart was isolated and placed under a dissecting microscope. Attached vessels and both atria were dissected and removed. The right ventricular wall was cut out, blotted, and weighed; then the left ventricular wall and septum were treated the same way and weighed.

\section{Measurement of plasma NO metabolites}

After anesthesia and injection with Heparin (250 units i.p.), mouse blood was collected by direct cardiac puncture. The plasma total NO was quantitatively determined based on the enzymatic conversion of nitrate to nitrite by nitrate reductase according with the manufactures instructions and as described previously. ${ }^{18}$

\section{Tissue histology}

Lungs from untreated and L-NAME treated animals were fixed and stained with hematoxylin and eosin, essentially as described elsewhere. ${ }^{13}$ All images were taken using a computerized analyzing system (Leica DMRB microscope, Wetzlar, Germany; Sony Power HAD camera, Tokyo, Japan).

\section{Assessment of exercise tolerance}

A 4-liter beaker filled with water $\left(34^{\circ} \mathrm{C}\right)$ was used as a swimming pool to assess the exercise tolerance of mice. The mouse was gently placed in the water and carefully observed. The time at which the mouse was initially unable to maintain complete buoyancy was recorded, and the mouse was immediately removed from the pool. No mice were injured in these experiments.

\section{Measurement of superoxide production}

Superoxide production was measured using the superoxide dismutase-inhibitable (SODinhibitable) cytochrome $c$ reduction assay. Animals were sacrificed by cervical dislocation. 
Wunderlich et al. NOS-inhibition prevents lung remodelling in caveolin-1 knockout mice

Aortas, pulmonary arteries and lungs were rapidly removed, cleaned of adventitial tissue and placed into chilled modified Krebs-buffer (composition in mmol/1: $117.48 \mathrm{NaCl}, 4.74 \mathrm{KCl}$, 2.50 $\mathrm{CaCl}_{2}, 1.19 \mathrm{MgSO}_{4}, 1.19 \mathrm{KH}_{2} \mathrm{PO}_{4}, 24.76 \mathrm{NaHCO}_{3}$ and 10 glucose [pH 7.4]). After cyotchrom c (50 $\mu$ M; SIGMA C-4186, Sigma-Aldrich, St. Louis, Missouri, USA) were added, the samples were incubated at $37^{\circ} \mathrm{C}$ for 60 minutes with and without SOD $(125 \mathrm{U} / \mathrm{ml})$. Cytochrome $c$ reduction was calculated using absorbance at $550 \mathrm{~nm}$ corrected for background readings at 540 and $560 \mathrm{~nm}$. Superoxide production was quantified in picomoles per milligram of the respective tissue (dry weight) from the difference between absorbance with or without SOD.

\section{Materials}

All compounds used in this study were either analytical grade or of the highest purity available. Heparin was obtained from ratiopharm (Ulm, Germany), Ketamin from CuraMED Pharma (Karlsruhe, Germany) and xylazine from medistar (Holzwickede, Germany). All other reagents were obtained from Merck (Darmstadt, Germany).

\section{Data Analysis}

All results are expressed as means \pm S.D. For multiple comparisons, ANOVA was applied, followed by the Bonferroni correction. Differences were assumed to be significant with a probability value of less than 0.05 .

\section{Results}

\section{Lowered systemic nitric oxide in L-NAME treated cav-1 ko}

Previous studies have unequivocally shown that cav-1 can bind and negatively regulate eNOS activity. We therefore assayed systemic levels the two major oxidation products of nitric oxide (NO), nitrate and nitrite $\left(=\mathrm{NO}_{\mathrm{x}}\right)$ in untreated and treated mice of both strains. Systemic $\mathrm{NO}_{\mathrm{x}}$ 
Wunderlich et al. NOS-inhibition prevents lung remodelling in caveolin-1 knockout mice

is generally regarded as a surrogate marker of systemic NO levels. In plasma obtained from untreated cav-1 ko $\mathrm{NO}_{\mathrm{x}}$ concentration was significantly higher than in WT mice (cav-1 ko: $8.85 \pm 1.43$ vs. $\underline{\mathrm{WT}}: 3.99 \pm 0.77 \mu \mathrm{M}, \mathrm{n}=10, \mathrm{P}<0.01)$. Several groups have recently shown by western blot analysis that no overt change in expression levels of the three major NO synthases (i.e., inducible, endothelial and neuronal NO synthases) was evident in cav-1 ko as compared to WT animals. Particularly, the inducible NO synthase was undetectable in cav-1 ko. ${ }^{9 ; 13}$ Given the close regulatory interaction between cav-1 and eNOS these findings suggest that the high $\mathrm{NO}_{\mathrm{x}}$ levels are most likely caused by an elevated eNOS activity in cav-1 ko. After L-NAME application systemic $\mathrm{NO}_{\mathrm{x}}$ levels fell significantly in both strains (cav-1 ko: $3.81 \pm 1.33$ vs. $\underline{\text { WT: }} 2.31 \pm 0.86 \mu \mathrm{M}, \mathrm{n}=10, \mathrm{P}<0.01$ vs. untreated). Notably, $\mathrm{NO}_{\mathrm{x}}$ concentration in L-NAME treated cav-1 ko approached the level of untreated WT mice.

\section{Normalized lung structure in L-NAME treated cav-1 ko}

One prominent feature of cav-1 ko is the adverse lung phenotype with thickened alveolar septa, smaller alveolar spaces and an interstitial hypercellularity. ${ }^{2 ; 3}$ All these findings are highly reminiscent of a human disorder named lung fibrosis. ${ }^{1}$ Given the hyperactivated NO pathway in cav-1 ko we studied the influence of chronic NOS inhibition by L-NAME on lung structure of cav-1 ko. In L-NAME-treated cav-1 ko we observed improvements of the lung architecture since the alveolar spaces appeared significantly wider and alveolar septa were thinner as compared with untreated cav-1 ko (Figure $1 \mathrm{C}+\mathrm{D})$. These findings are further corroborated by marked reductions in lung weight to body weight ratios in L-NAME treated cav-1 ko (Figure 2 A). No differences were evident between untreated and L-NAME treated WT mice. 
Wunderlich et al. NOS-inhibition prevents lung remodelling in caveolin-1 knockout mice

\section{Improved lung function in L-NAME treated cav-1 ko}

In view of the adverse lung fibrosis in cav-1 ko we looked in detail on the oxygen saturation with and without L-NAME administration. Without chronic NOS inhibition cav-1 ko displayed a significantly reduced oxygen saturation which was completely normalized after LNAME treatment (Table 1). As compared to WT littermates hemoglobin and hematocrit levels revealed a polyglobic blood count in untreated cav-1 ko which is most probably a sequel of hypoxic erythropoetin stimulation. As depicted in Table 1 L-NAME application substantially lowered these pathological values although no complete normalization was achieved.

\section{Reversal of the pulmonary hypertension, right ventricular hypertrophy and right ventricular heart failure in L-NAME treated cav-1 ko}

Cav-1 ko are additionally characterized by markedly hypertrophied right ventricles (RV). The $\mathrm{RV}$ to body weight ratio was significantly increased in untreated cav-1 ko $(1.44 \pm 0.2, \mathrm{n}=10)$ as compared to WT littermates $(1.06 \pm 0.2, \mathrm{n}=12, \mathrm{P}<0.05)$ and was accompanied by a profound pulmonary hypertension (Figure $3 \mathrm{~A}$ ). The left ventricular end diastolic pressure (LVEDP) was elevated in untreated cav-1 ko $(15.5 \pm 1.9 \mathrm{mmHg})$ as compared to WT mice $(9.0 \pm 1.9$ $\mathrm{mmHg}, \mathrm{n}=10-12, \mathrm{P}<0.05$ ). The same was true for the transpulmonary gradient (TPG - Figure $3 \mathrm{~B}$ ). Of note, the left ventricular (LV) to RV ratio was dramatically reduced (Figure $2 \mathrm{~B}$ ) and similar to that seen in other pulmonary hypertension models. Additionally, the central venous pressure was significantly elevated in cav-1 ko as compared to WT counterparts (cav-1 ko: $17.4 \pm 3.5$ vs. $\underline{\mathrm{WT}}: 4.36 \pm 2.4 \mathrm{mmHg}, \mathrm{n}=7, \mathrm{P}<0.01)$. The liver to body weight ratio was dramatically increased in cav-1 ko as compared to WT mice (cav-1 ko: $57.77 \pm 5.52$ vs. WT: $44.36 \pm 5.65 \mathrm{mg} / \mathrm{g}, \mathrm{n}=8-12, \mathrm{P}<0.01)$.

In view of these findings we studied the influence of L-NAME treatment on these distinct parameters. While the RV to body weight ratio in L-NAME treated cav-1 ko significantly 
Wunderlich et al. NOS-inhibition prevents lung remodelling in caveolin-1 knockout mice

decreased $(0.96 \pm 0.1, \mathrm{n}=9, \mathrm{P}<0.05$ vs. untreated cav-1-ko) it did not change in L-NAME treated WT animals $(0.94 \pm 0.04, \mathrm{n}=13)$. As shown in Figure 2 B L-NAME donation in cav-1 ko resulted in an increased LV/RV ratio. Furthermore, after L-NAME treatment LVEDP decreased in both strains mice (cav-1 ko: $6.8 \pm 1.9 \mathrm{P}<0.01$ vs. untreated cav-1 ${ }^{-/-}, \underline{\text { WT: }} 7.0 \pm 2.8$ mmHg, $\mathrm{n}=8-12$ ). Likewise, as depicted in Figure 3, we observed a reduction of both the pulmonary artery hypertension and the TPG after chronic NOS inhibition with L-NAME. As a sign of the reduced right ventricular congestion both the central venous pressure (cav-1 ko: 7.2 $\pm 4.3 \mathrm{P}<0.01$ vs. untreated cav-1 ko, WT: $5.7 \pm 3.6 \mathrm{mmHg}, \mathrm{n}=6, \mathrm{P}<0.01)$ and the liver/body weight ratio dramatically decreased after L-NAME treatment (cav-1 ko: $52.22 \pm 2.26 \mathrm{P}<0.01$ vs. untreated cav-1 $1^{-/}$, WT: $\left.46.37 \pm 1.54 \mathrm{mg} / \mathrm{g}, \mathrm{n}=8-12\right)$.

\section{L-NAME treatment in cav-1 ko results in improved exercise capacity}

Cav-1 ko have been found to exhibit a profound exercise intolerance. ${ }^{3}$ This observation may be attributed to the severe lung fibrosis and the pulmonary arterial hypertension. Since we observed substantial regressions in both findings after L-NAME administration we grossly assessed the possible physical consequences of L-NAME treatment by subjecting these animals to a swimming test. In this way, we could directly compare the exercise tolerance of WT, cav-1 ko, and L-NAME treated mice of both strains. As displayed in figure 4 WT animals were able to swim for up to $35 \mathrm{~min}$, while cav-1 ko were exhausted after $13 \mathrm{~min}$. This is contrasted by significant improvements in the exercise capacity of L-NAME treated cav-1 ko (Figure 4).

\section{Enhanced superoxide generation in cav-1 ko is reduced after L-NAME treatment}

A possible explanation for the adverse pulmonary phenotype is enhanced oxidant stress in cav-1 ko. To test this hypothesis we harvested aortas, pulmonary arteries and lung tissue from untreated animals and performed a cytochrome $c$ reduction assay for measurement of $\mathrm{O}_{2}{ }^{-}$ 
Wunderlich et al. NOS-inhibition prevents lung remodelling in caveolin-1 knockout mice

release. We observed striking disparities between WT and cav-1 ko in aorta, pulmonary artery (data not shown, but almost identical as compared to aortic tissue) and lung tissue with a markedly increased $\mathrm{O}_{2}^{-}$production in knockout animals (Figure 5 A, B). Given the aforementioned hyperactivity of the eNOS-NO pathway in cav-1 ko we next treated animals of both strains with oral L-NAME and measured ROS generation in these animals. As shown in Figure 5 L-NAME-supplementation resulted in a decreased $\mathrm{O}_{2}^{-}$production in lungs and aortas taken from cav-1 ko.

\section{Discussion}

Several groups have independently generated cav-1 ko, and these animals were repeatedly shown to suffer from clear and reproducible vascular and pulmonary phenotypes including a severe lung fibrosis with pulmonary arterial hypertension (PAH). ${ }^{2 ; 3}$ These phenotypes, driven by the global loss of cav-1, may be caused by the deficiency of the gene in cells that normally express this protein, including endothelial cells, adipocytes, fibroblasts, epithelial cells and smooth muscle cells. A plethora of biochemical and genetic data support the role of cav-1 as a negative regulator of agonist-mediated eNOS activation. 3;79;13 Accordingly, constitutive eNOS hyperactivation was observed in cav-1 ko. The present study argues that major lung pathologies are caused at least in part by dysregulated NO synthases resulting in enhanced oxidative stress since chronic treatment with the NO synthase inhibitor L-NAME prevented the evolution of the pulmonary abnormalities and alleviated the oxidative stress in cav-1 ko. These findings are inasmuch of paramount importance since they underline the central role of a balanced NO signalling in normal pulmonary physiology.

Recent insights into the physiological roles of caveolae and caveolins have been dissected in genetically modified mice. It was revealed that cav-1 is essential for the formation of caveolae in fibroblasts, endothelial cells, adipocytes. ${ }^{2 ; 3}$ Additionally, it was shown that the loss of cav1 , which is the primary isoform in the vascular system, results in multiple alterations with a 
Wunderlich et al. NOS-inhibition prevents lung remodelling in caveolin-1 knockout mice

dysregulation in NO synthesis ${ }^{3 ; 9}$, vascular permeability $^{6}$, mechanotransduction $^{19}$ and pulmonary function. ${ }^{9}$ These studies unequivocally validate the in vivo importance of cav-1 beyond cells based studies which are hampered by operational definitions of biochemical fractions containing caveolins and the lack of specificity inherent in reagents that extract cholesterol from cells. ${ }^{6}$

PAH is a common finding in hypoxic lung disorders like lung fibrosis. Pulmonary hypertension arises when vasoconstriction and adverse remodelling of pulmonary arterioles results in increased pulmonary vascular resistance. The subsequent rise in pressure load promotes right ventricular hypertrophy, which often progresses to premature death from right heart failure. ${ }^{17}$ Cav-1 has been suggested to function as a key regulator of adverse lung remodelling and the development of $\mathrm{PAH}^{5-8}$ Indeed, cav-1 ko were shown to suffer from a severe lung fibrosis with thickened alveolar septa, smaller alveolar spaces and an interstitial hypercellularity. ${ }^{2 ; 3}$ Our findings demonstrate that loss of cav-1 in lung parenchyma also results in PAH and right ventricular hypertrophy which validates former findings. ${ }^{9}$ All these features are highly reminiscent of the human lung fibrosis which is frequently associated with PAH. Moreover, we found signs of right ventricular heart failure as evidenced by dramatically increased liver to body weight ratio in cav-1 ko. To the best of our knowledge this study is the first to show that cav-1 ko additionally display a significantly reduced arterial oxygen saturation accompanied by a polyglobic blood count with increased hemoglobin and hematocrit values. Elevated hemoglobin and hematocrit levels are a common finding in chronic lung disorders and are regarded as a sequel of enhanced hypoxic erythropoetin stimulation. Given these characteristics evident in untreated cav-1 ko these animals may be viewed as an useful model of PAH in lung fibrosis.

In normal lung tissue, endothelial cells and type I pneumocytes, abundantly express cav-1. Therefore it seems easily conceivable that histological and functional abnormalities develop in lungs of cav-1 ko. Nevertheless the specific role of cav-1 and caveolae in this process is 
Wunderlich et al. NOS-inhibition prevents lung remodelling in caveolin-1 knockout mice

still a matter of discussion. Cav-1 is also regarded as a candidate tumor suppressor. In line with this notion, Razani et al. found dramatically increased markers of cellular proliferation in lungs of cav-1 ko. ${ }^{2}$ Therefore they suggested that disruption of cav-1 leads to profound aberrations in cells cycle checkpoints resulting in the abovementioned pathologies.

In view of the close interaction of cav-1 and eNOS in normal physiology we questioned whether a perturbation in NO signalling is involved in adverse lung remodelling in cav-1 ko. There is mounting evidence that validates the negative regulatory influence of cav-1 on eNOS function..$^{3 ; 79 ; 13}$ For instance, enhanced NO-mediated vascular relaxation in isolated aortic rings ${ }^{3}$ together with increased systemic $\mathrm{NO}_{\mathrm{x}}$ levels ${ }^{9}$ and enhanced eNOS activity in vitro was reported. ${ }^{13}$ Since it was repeatedly demonstrated by western blots that no change in expression level of the major NO synthases (i.e., eNOS, inducible NOS, neuronal NOS) in vessels and lungs of cav-1 ko occurred ${ }^{7 ; 9 ; 13}$ these findings suggest that the high NO levels we and others have measured are most likely caused by an elevated eNOS activity. Since eNOS derived NO, through potent vasodilator and antiproliferative effects on vascular smooth muscle cells is critical in maintaining normal pulmonary vascular tone and structure it seems difficult to relate eNOS activity to the adverse pulmonary phenotype of cav-1 ko. But accumulating evidence indicates that in some circumstances eNOS can be converted into a important source of radicals within the vessel wall. ${ }^{14 ; 15}$ In line with this concept we measured a markedly increased $\mathrm{O}_{2}^{-}$generation in vessels and lung tissue harvested from untreated cav-1 ko. The biological sequelae of the so-called eNOS "uncoupling" on NO bioavailability may be profound, because NO production is reduced and $\mathrm{O}_{2}^{-}$generation is increased. Since superoxide is known to act as a potent scavenger of bioactive NO this scenario favours significant reductions in the bioavailability of vasodilating NO. Of note, increased $\mathrm{O}_{2}{ }^{-}$ generation has been observed in other experimental models of $\mathrm{PAH}^{20}$ and markers of oxidative stress are increased in patients with $\mathrm{PAH} .{ }^{17}$ Superoxide mediates pulmonary vasoconstriction and stimulates pulmonary smooth muscle proliferation. ${ }^{21}$ In view of our data 
Wunderlich et al. NOS-inhibition prevents lung remodelling in caveolin-1 knockout mice

it is tempting to speculate that L-NAME donation resulted in a decreased $\mathrm{O}_{2}{ }^{-}$generation by dysfunctional eNOS in the pulmonary endothelium thereby preventing the detrimental effects of $\mathrm{O}_{2}^{-}$on pulmonary smooth muscle cells and NO bioavailability. Notably, a recent paper reports that a reduction in oxidative stress by gene transfer of extracellular SOD results in a amelioration of $\mathrm{PAH}$ in rat model. ${ }^{22}$

In agreement with this concept we could additionally demonstrate that chronic NOS inhibition resulted in a reduced $\mathrm{O}_{2}^{-}$generation in cav-1 ko and prevented adverse lung remodelling in treated knockout animals. Improvements of the lung architecture with wider alveolar spaces and thinner alveolar septa (Figure 1 A-D) were accompanied by an improved lung function as demonstrated by normalized oxygen saturation and improved blood cell count in treated knockout animals. Moreover, we observed a normalisation of the pulmonary artery pressure in conjunction with an improved blood oxygenation and a regression of both the right ventricular hypertrophy and right ventricular heart failure in L-NAME treated cav-1 ko. Moreover, LNAME treatment resulted in an enhanced exercise capacity.

The pathogenesis of PAH is complex and involves a blend of structural, functional and biological changes leading to alterations in the pulmonary circulation. In cav-1 ko PAH could be caused by the interstitial lung disease due to cav-1 ablation or it could be secondary to left ventricular impairment. Although we and others observed signs of left ventricular dysfunction the pronounced increase in the TPG in cav-1 ko indicates that intrinsic defects of the pulmonary vessels leading to enhanced vasoconstriction is at least partially involved in the development of PAH. Indeed, recent advances in the understanding of the molecular mechanism involved in the evolution of human PAH suggest endothelial dysfunction as a hallmark of the disease. Impaired production of vasodilating mediators, such as nitric oxide and prostacyclin, along with sustained overexpression of vasoconstrictors like endothelin-1 promote both vasoconstriction and vascular remodelling. Thus, these substances have emerged as important targets of pharmacological therapy of PAH in humans. ${ }^{17}$ 
Wunderlich et al. NOS-inhibition prevents lung remodelling in caveolin-1 knockout mice

In summary, we suggest a pivotal role of dysfunctional NO signalling in the pathogenesis of the lung fibrosis and severe pulmonary arterial hypertension in cav-1 ko. The present study underlines the utmost importance of a balanced cav-1 eNOS interaction for normal lung physiology and demonstrate that perturbations of normal NOS function may have devastating consequences on lung function. Whether the same holds true in human disorders remains to be established in future studies. However, such a perspective would open a completely new therapeutic strategy to pulmonary diseases. 
Wunderlich et al. NOS-inhibition prevents lung remodelling in caveolin-1 knockout mice

\section{Figure legends}

Figure 1. The pronounced pathomorphological lung defects of cav-1 ko are prevented by chronic L-NAME treatment. Representative paraffin sections of lung tissue from WT $(\mathbf{A}, \mathbf{B})$ and caveolin-1 deficient mice $(\mathbf{C}, \mathbf{D})$ were stained with hematoxylin-eosin without and after L-NAME treatment.

Assignments: WT, wild type mice; cav-1 ko, caveolin-1 knockout mice; L-NAME - $\mathrm{N}^{\mathrm{G}}$-nitroL-arginine methyl ester.

Figure 2. The beneficial effects of chronic L-NAME treatment on lung structure are further corroborated by a reduced lung weight to body weight ratio in L-NAME treated cav-1 ko (A). Additionally, L-NAME donation prevented the development of the substantial right ventricular hypertrophy in cav-1 ko (B).

Assignments: WT, wild type mice; cav-1 ko, caveolin-1 knockout mice; L-NAME - $\mathrm{N}^{\mathrm{G}}$-nitroL-arginine methyl ester, Symbols show means $\pm \mathrm{SD}$ for $\mathrm{n}=10-12 ; * P<0.01$ vs. wild type. ** $P<0.01$ vs. untreated cav-1 ko.

Figure 3. In vivo cardiac catheterization revealed a marked pulmonary hypertension in untreated cav-1 ko which was significantly alleviated by chronic L-NAME donation (A). The marked increase in the TPG in untreated cav-1 ko indicates that intrinsic defects of the pulmonary vessels with enhanced pulmonary vasoconstriction playing a major role in pulmonary hypertension of untreated cav-1 ko. Chronic NO synthases inhibition markedly reduced the TPG. $(\mathbf{B})$.

Assignments: WT, wild type mice; cav-1 ko, caveolin-1 knockout mice; L-NAME - $\mathrm{N}^{\mathrm{G}}$-nitroL-arginine methyl ester, TPG, transpulmonary gradient; Symbols show means \pm SD for $n=$ $10-12 ; * P<0.01$ vs. wild type. ${ }^{* *} P<0.01$ vs. untreated caveolin-1 knockout mice. 
Wunderlich et al. NOS-inhibition prevents lung remodelling in caveolin-1 knockout mice

Figure 4. As shown by swimming chamber tests untreated cav-1 ko display a physical disability which was markedly improved after chronic L-NAME donation.

Assignments: WT, wild type mice; cav-1 ko, caveolin-1 knockout mice; L-NAME - $\mathrm{N}^{\mathrm{G}}$-nitroL-arginine methyl ester, Symbols show means \pm SD for $n=6-8 ; * P<0.01$ vs. wild type. ** $P<0.01$ vs. untreated caveolin-1 knockout mice.

Figure 5. The enhanced oxidative stress in lung tissue and aortas (as estimated by SOD inhibitable cytochrom c reduction assay) in untreated cav-1 ko is markedly diminished after L-NAME donation.

Assignments: WT, wild type mice; cav-1 ko, caveolin-1 knockout mice; L-NAME - $\mathrm{N}^{\mathrm{G}}$-nitroL-arginine methyl ester, Symbols show means $\pm \mathrm{SD}$ for $\mathrm{n}=6-8 ; * P<0.01$ vs. wild type. ** $P<0.01$ vs. untreated caveolin-1 knockout mice.

Table 1. Oxygen saturation, hemoglobin and hematocrit level in untreated and L-NAMEtreated WT and cav-1 ko. The improved oxygen saturation and the normalized hemoglobin and hematocrit values in L-NAME treated cav-1 ko indicate a markedly improved lung function after chronic NO synthase inhibition.

Assignments: cav-1 ko, caveolin-1 knockout mice; L-NAME, L-NAME - $\mathrm{N}^{\mathrm{G}}$-nitro-L-arginine methyl ester, Symbols show means \pm SD. 
Wunderlich et al. NOS-inhibition prevents lung remodelling in caveolin-1 knockout mice

\section{Reference List}

1. Razani, B. and M. P. Lisanti. 2001. Caveolin-deficient mice: insights into caveolar function human disease. J.Clin.Invest 108:1553-1561.

2. Razani, B., J. A. Engelman, X. B. Wang, W. Schubert, X. L. Zhang, C. B. Marks, F. Macaluso, R. G. Russell, M. Li, R. G. Pestell, D. Di Vizio, H. Hou, Jr., B. Kneitz, G. Lagaud, G. J. Christ, W. Edelmann, and M. P. Lisanti. 2001. Caveolin-1 null mice are viable but show evidence of hyperproliferative and vascular abnormalities. J.Biol.Chem. 276:38121-38138.

3. Drab, M., P. Verkade, M. Elger, M. Kasper, M. Lohn, B. Lauterbach, J. Menne, C. Lindschau, F. Mende, F. C. Luft, A. Schedl, H. Haller, and T. V. Kurzchalia. 2001. Loss of caveolae, vascular dysfunction, and pulmonary defects in caveolin-1 gene-disrupted mice. Science 293:2449-2452.

4. Galbiati, F., J. A. Engelman, D. Volonte, X. L. Zhang, C. Minetti, M. Li, H. Hou, Jr., B. Kneitz, W. Edelmann, and M. P. Lisanti. 2001. Caveolin-3 null mice show a loss of caveolae, changes in the microdomain distribution of the dystrophin-glycoprotein complex, and t-tubule abnormalities. J.Biol.Chem. 276:21425-21433.

5. Razani, B., X. B. Wang, J. A. Engelman, M. Battista, G. Lagaud, X. L. Zhang, B. Kneitz, H. Hou, Jr., G. J. Christ, W. Edelmann, and M. P. Lisanti. 2002. Caveolin-2deficient mice show evidence of severe pulmonary dysfunction without disruption of caveolae. Mol.Cell Biol. 22:2329-2344.

6. Gratton, J. P., P. Bernatchez, and W. C. Sessa. 2004. Caveolae and caveolins in the cardiovascular system. Circ.Res. 94:1408-1417. 
Wunderlich et al. NOS-inhibition prevents lung remodelling in caveolin-1 knockout mice

7. Murata, T., M. I. Lin, Y. Huang, J. Yu, P. M. Bauer, F. J. Giordano, and W. C. Sessa. 2007. Reexpression of caveolin-1 in endothelium rescues the vascular, cardiac, and pulmonary defects in global caveolin-1 knockout mice. J.Exp.Med. 204:2373-2382.

8. Jasmin, J. F., I. Mercier, R. Hnasko, M. W. Cheung, H. B. Tanowitz, J. Dupuis, and M. P. Lisanti. 2004. Lung remodeling and pulmonary hypertension after myocardial infarction: pathogenic role of reduced caveolin expression. Cardiovasc.Res. 63:747-755.

9. Zhao, Y. Y., Y. Liu, R. V. Stan, L. Fan, Y. Gu, N. Dalton, P. H. Chu, K. Peterson, J. Ross, Jr., and K. R. Chien. 2002. Defects in caveolin-1 cause dilated cardiomyopathy and pulmonary hypertension in knockout mice. Proc.Natl.Acad.Sci.U.S.A 99:1137511380.

10. Park, D. S., A. W. Cohen, P. G. Frank, B. Razani, H. Lee, T. M. Williams, M. Chandra, J. Shirani, A. P. De Souza, B. Tang, L. A. Jelicks, S. M. Factor, L. M. Weiss, H. B. Tanowitz, and M. P. Lisanti. 2003. Caveolin-1 null (-/-) mice show dramatic reductions in life span. Biochemistry 42:15124-15131.

11. Mathew, R., J. Huang, M. Shah, K. Patel, M. Gewitz, and P. B. Sehgal. 2004. Disruption of endothelial-cell caveolin-1alpha/raft scaffolding during development of monocrotaline-induced pulmonary hypertension. Circulation 110:1499-1506.

12. Achcar, R. O., Y. Demura, P. R. Rai, L. Taraseviciene-Stewart, M. Kasper, N. F. Voelkel, and C. D. Cool. 2006. Loss of caveolin and heme oxygenase expression in severe pulmonary hypertension. Chest 129:696-705. 
Wunderlich et al. NOS-inhibition prevents lung remodelling in caveolin-1 knockout mice

13. Wunderlich, C., K. Schober, S. A. Lange, M. Drab, R. C. Braun-Dullaeus, M. Kasper, C. Schwencke, A. Schmeisser, and R. H. Strasser. 2006. Disruption of caveolin-1 leads to enhanced nitrosative stress and severe systolic and diastolic heart failure. Biochem.Biophys.Res.Commun. 340:702-708.

14. Kuzkaya, N., N. Weissmann, D. G. Harrison, and S. Dikalov. 2003. Interactions of peroxynitrite, tetrahydrobiopterin, ascorbic acid, and thiols: implications for uncoupling endothelial nitric-oxide synthase. J.Biol.Chem. 278:22546-22554.

15. Xia, Y., V. L. Dawson, T. M. Dawson, S. H. Snyder, and J. L. Zweier. 1996. Nitric oxide synthase generates superoxide and nitric oxide in arginine-depleted cells leading to peroxynitrite-mediated cellular injury. Proc.Natl.Acad.Sci.U.S.A 93:6770-6774.

16. Szabo, C., H. Ischiropoulos, and R. Radi. 2007. Peroxynitrite: biochemistry, pathophysiology and development of therapeutics. Nat.Rev.Drug Discov. 6:662-680.

17. Humbert, M., O. Sitbon, and G. Simonneau. 2004. Treatment of pulmonary arterial hypertension. N.Engl.J.Med. 351:1425-1436.

18. Wunderlich, C., U. Flogel, A. Godecke, J. Heger, and J. Schrader. 2003. Acute inhibition of myoglobin impairs contractility and energy state of iNOS-overexpressing hearts. Circ.Res. 92:1352-1358.

19. Yu, J., S. Bergaya, T. Murata, I. F. Alp, M. P. Bauer, M. I. Lin, M. Drab, T. V. Kurzchalia, R. V. Stan, and W. C. Sessa. 2006. Direct evidence for the role of caveolin1 and caveolae in mechanotransduction and remodeling of blood vessels. J.Clin.Invest 116:1284-1291. 
Wunderlich et al. NOS-inhibition prevents lung remodelling in caveolin-1 knockout mice

20. Brennan, L. A., R. H. Steinhorn, S. Wedgwood, E. Mata-Greenwood, E. A. Roark, J. A. Russell, and S. M. Black. 2003. Increased superoxide generation is associated with pulmonary hypertension in fetal lambs: a role for NADPH oxidase. Circ.Res. 92:683691.

21. Wedgwood, S. and S. M. Black. 2003. Role of reactive oxygen species in vascular remodeling associated with pulmonary hypertension. Antioxid.Redox.Signal. 5:759-769.

22. Kamezaki, F., H. Tasaki, K. Yamashita, M. Tsutsui, S. Koide, S. Nakata, A. Tanimoto, M. Okazaki, Y. Sasaguri, T. Adachi, and Y. Otsuji. 2007. Gene Transfer of Extracellular Superoxide Dismutase Ameliorates Pulmonary Hypertension in Rats. Am.J.Respir.Crit Care Med. Epub ahead of print 
Figure 2
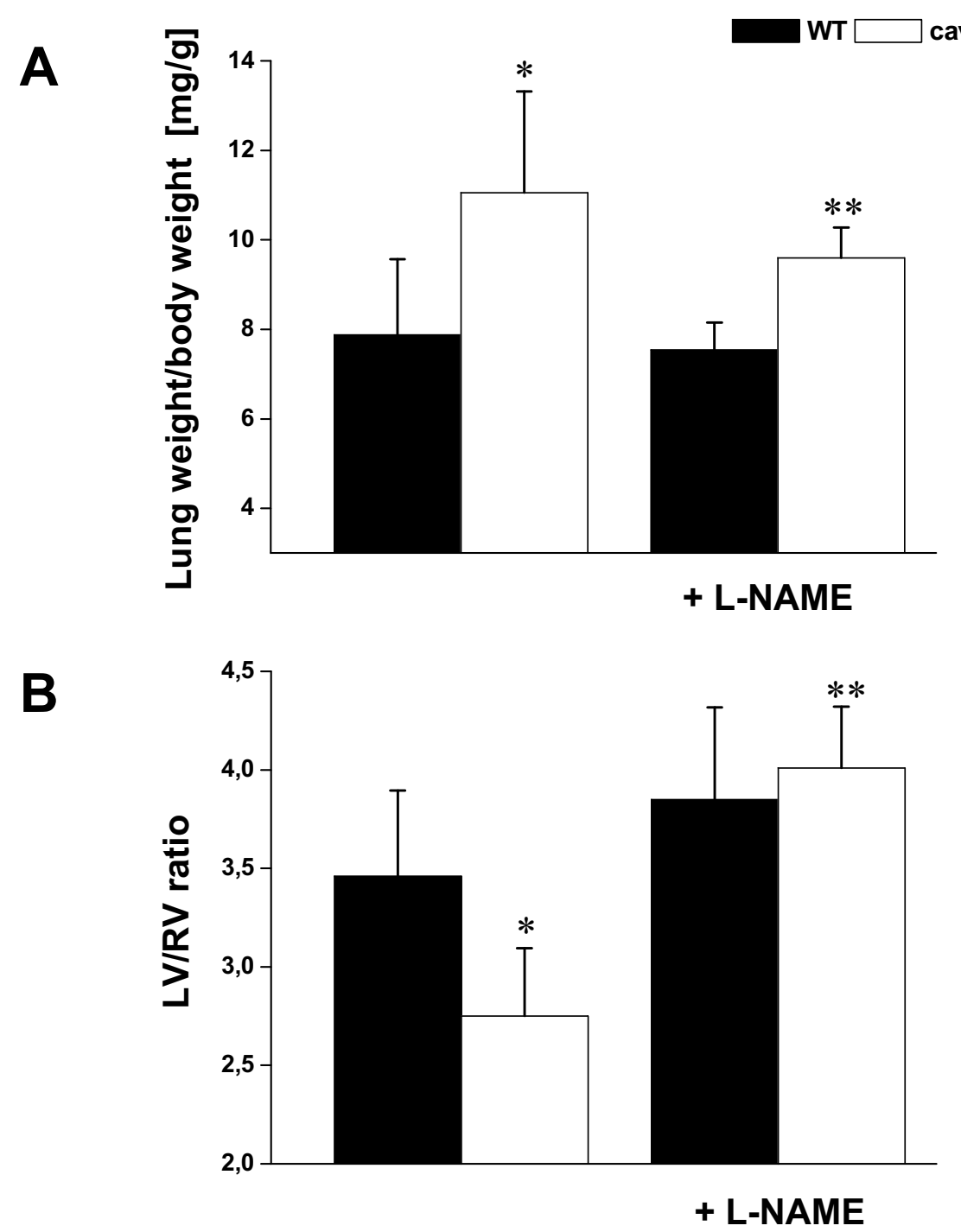


\section{Table 1}

\begin{tabular}{|c|c|c|c|c|}
\hline & & $\begin{array}{c}\text { O2 saturation } \\
{[\%]}\end{array}$ & $\begin{array}{c}\text { hemoglobin } \\
\text { [mmol/l] }\end{array}$ & $\begin{array}{c}\text { hematocrit } \\
{[\%]}\end{array}$ \\
\hline & $\begin{array}{l}\text { wild type } \\
n=14\end{array}$ & $98.5 \pm 0.3$ & $8.6 \pm 0.3$ & $43.8 \pm 2.4$ \\
\hline & $\underset{n=10}{\operatorname{cav}-1 \text { ko }}$ & $91.5 \pm 1.8$ * & $10.4 \pm 0.4$ * & $55.2 \pm 1.2$ * \\
\hline \multirow{2}{*}{ + L-NAME } & $\begin{array}{l}\text { wild type } \\
n=11\end{array}$ & $98.2 \pm 0.3$ & $7.9 \pm 0.7$ & $39.0 \pm 3.0$ \\
\hline & $\begin{array}{c}\text { cav-1 ko } \\
n=12\end{array}$ & $98.0 \pm 2.31$ ** & $9.3 \pm 1.7$ ** & $49.0 \pm 3.0$ ** \\
\hline
\end{tabular}

${ }^{\star} P<0.05$ vs. wild type, ${ }^{* *} P<0.05$ vs. untreated cav-1 ko 
Figure 3
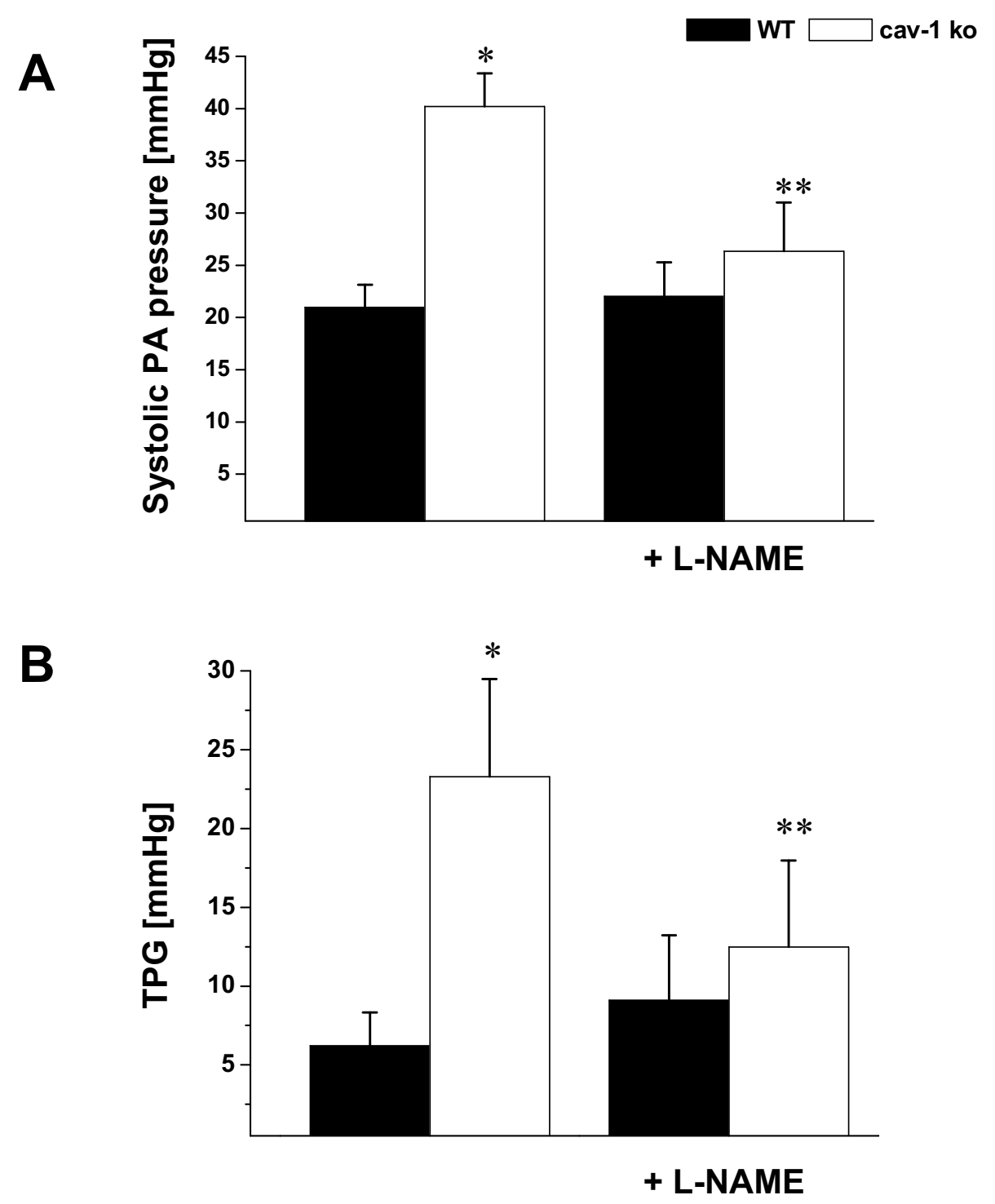
Figure 4

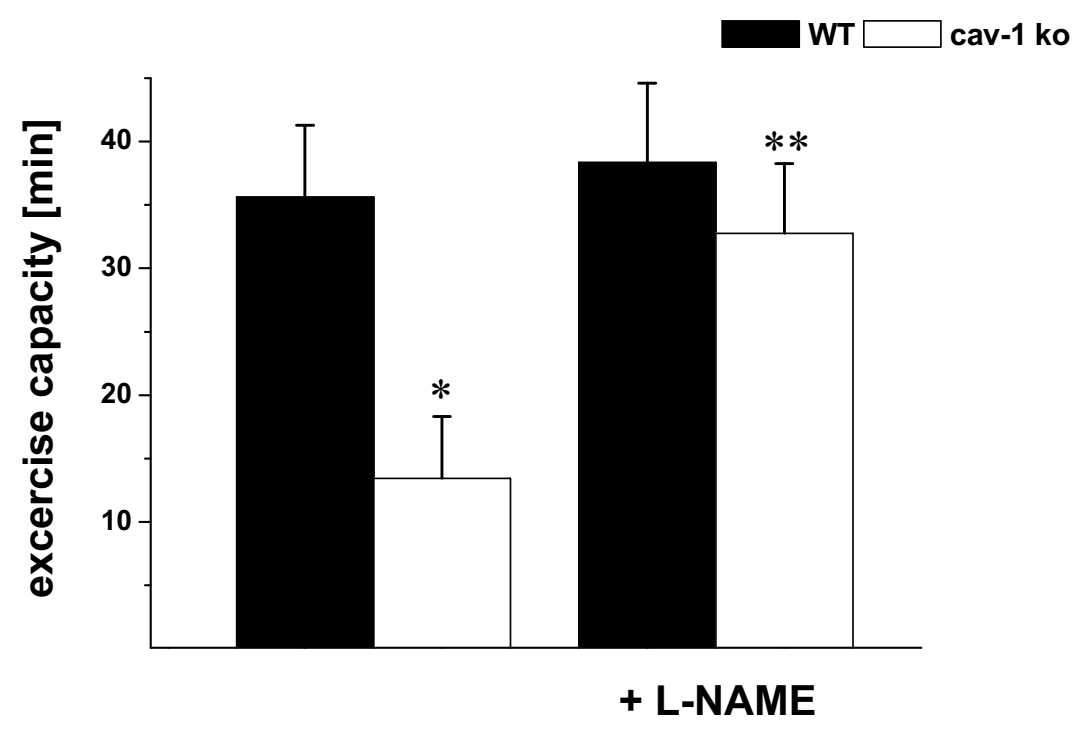


Figure 5

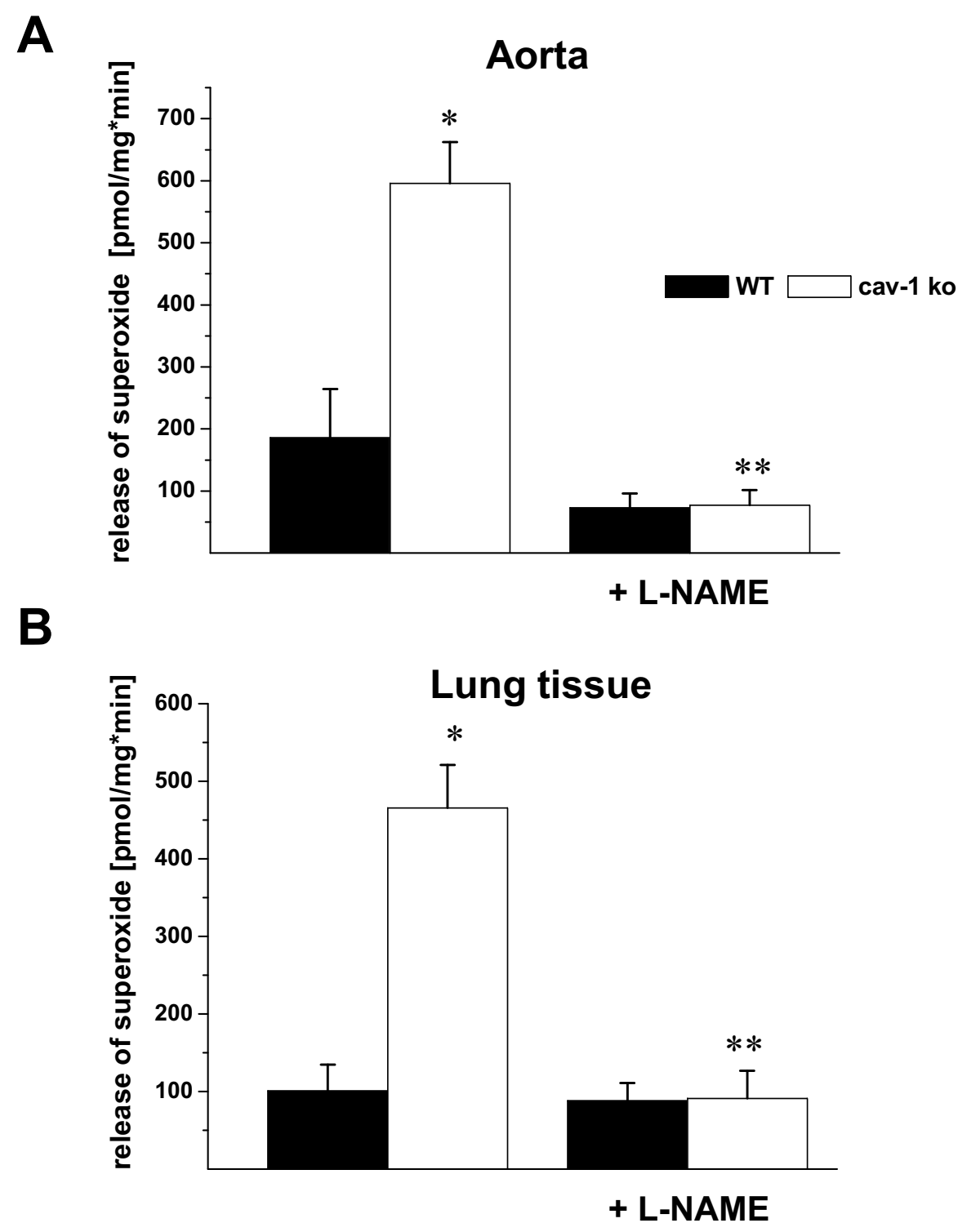

